\title{
Objetivo Madrid: planes de ocupación y concepción del orden público durante la Guerra Civil española
}

\author{
Alejandro Pérez-Olivares García \\ Seminario Complutense "Historia, Cultura y Memoria"/ Departamento de Historia Contemporánea, Universidad Complutense de Madrid. \\ Facultad de Geografía e Historia, Edificio B. Calle Profesor Aranguren, s/n, 28040 (Madrid) \\ e-mail: aperezolivares@ucm.es
}

Submitted: 11 May 2015. Accepted: 25 July 2015

RESUMEN: El presente texto pretende ofrecer una nueva visión sobre la Guerra Civil y la violencia ejercida en su marco. Por un lado, trata de explicar la sublevación de 1936 como una reacción al proceso de Modernidad experimentado principalmente en la España urbana, y concretamente en Madrid. Por otro, procura adentrarse en la lógica funcional de la violencia en detrimento de los presupuestos exterministas dominantes en la historiografía española. Es necesario ampliar la secuencia explicativa II República-sublevación-guerra para comprender el 18 de julio desde unos parámetros más dilatados, que proyectan las expresiones de la violencia durante el conflicto hacia la posguerra a través de la gestión del orden público. Mediante el Servicio de Recuperación de Documentos y del Cuartel General del Generalísimo se documenta el plan de ocupación para Madrid. Una fuente sin duda novedosa para comprender la concepción franquista de lo que debía ser el espacio público y comprobar cómo se materializaron las bases sobre las que debía construirse el rechazo a los cambios producidos en el primer tercio del siglo xx, percibidos como una amenaza a los valores tradicionales.

PALABRAS CLAVE: Madrid; control social; guerra civil española; Franquismo; orden público; violencia política.

Citation / Cómo citar este artículo: Pérez-Olivares García, Alejandro (2015) "Objetivo Madrid: planes de ocupación y concepción del orden público durante la Guerra Civil española”. Culture \& History Digital Journal, 4(2): e019. doi: http:// dx.doi.org/10.3989/chdj.2015.019

\begin{abstract}
Target Madrid: plans for occupation and design of public order during the Spanish Civil War.- This article looks at Spanish Civil War and violence in those days from a different point of view. On the one hand, I try to explain the military coup of July 1936 as a reaction against the experience of Modernity in the spanish cities, focusing on Madrid. On the other, I try to go in depth into the functional logic of violence instead of the hegemonic historiographical proposals. It is necessary to explain the sequence 2nd Republic-Coup-War in the mid-long range to understand the significance of Francoist Public Order model. Using the occupation plan for Madrid by Franco's Headquarters and Servicio de Recuperación de Documentos paperwork, this article attempts to get the Dictatorship's view about public space and sociocultural transformations in the early decades of xxth Century.
\end{abstract}

KEYWORDS: Madrid; Social Control; Spanish Civil War; Francoism; Public Order; Political Violence.

Copyright: (C) 2015 CSIC This is an open-access article distributed under the terms of the Creative Commons AttributionNon Commercial (by-nc) Spain 3.0 License. 


\section{MADRID, 1936: UNA METRÓPOLIS MODERNA}

Cuando en noviembre de 1936 los sublevados se encontraban a las puertas de Madrid, la ciudad que se alzaba ante ellos era completamente diferente a las localidades que habían ocupado progresivamente desde el mes de julio. Los mandos eran conscientes de esta cualidad y repartieron entre las tropas la siguiente consigna, de obligado cumplimiento al entrar en la capital:

La ocupación de Madrid tiene que ser un ejemplo de disciplina y organización [...] Lo peligroso de la ocupación de una gran población y la necesidad de poder reaccionar en cualquier momento contra la sorpresa, impone que toda tropa se encuentre, en todo momento, en condiciones de combatir y que los mandos extremen las prevenciones para evitar toda sorpresa ${ }^{1}$.

La importancia de la capital para los sublevados resultaba innegable. Centro político, administrativo y financiero, desde los primeros momentos de la conspiración fue el principal objetivo militar de una operación que se pretendía rápida. Pero Madrid era algo más que la residencia de las principales instituciones del país. Desde finales del siglo XIX y en un proceso acelerado a partir de las dos primeras décadas del xx, Madrid se convirtió en una ciudad moderna, equiparable a Londres, París o Nueva York. Y no lo fue únicamente por el volumen de población que acogía (superó el millón de personas en 1930), su extensión (que alcanzaba a municipios colindantes como Chamartín de la Rosa, Tetuán o Vallecas sin solución de continuidad) o la progresiva disolución de las estructuras económicas heredadas del siglo anterior. La distancia entre el lugar de residencia y el trabajo se había ampliado progresivamente, un hecho favorecido por el desarrollo de modernos sistemas de transporte urbano como el tranvía o el metro. La estructura socioeconómica madrileña era más variada y compleja, puesto que los empleados de servicios coexistían con jornaleros proletarizados que trabajaban en la construcción, motor del crecimiento económico de la ciudad. En sus calles se instalaron modernas sociedades de inversión y centros comerciales que hacían uso de técnicas publicitarias importadas directamente de Estados Unidos y se construyeron recintos donde celebrar espectáculos deportivos que reunían a miles de personas (Otero Carvajal y Pallol Trigueros, 2009; Pallol Trigueros, 2009; Rodríguez Martín, 2013).

Madrid se había modernizado, vio cómo se aceleraban sus tiempos y se transformaba su espacio. Más aún cuando, durante la II República, las autoridades políticas promovieron la identificación de la ciudad con el régimen. Madrid, que ya era capital del libro por la industria editorial que concentraba, se convirtió durante los años 30 en el verdadero centro de la llamada "Edad de Plata" de la ciencia y cultura españolas. Reflejo de ello fueron las ferias del libro, los espacios dedicados al nuevo campus de la Universidad Central en Moncloa, las instalaciones de la Junta para Ampliación de Estudios en los alrededores del Paseo de la Castellana y los modernos ensayos peda- gógicos en torno al Retiro. Las autoridades locales y nacionales quisieron impulsar definitivamente un proceso, el de la transformación de los súbditos en ciudadanos, que corrió paralelo a la modificación de las coordenadas espaciales y mentales de la propia ciudad, pues los nuevos espacios destinados a la difusión de una cultura moderna coincidían con el eje Sur-Norte de proyección urbanística de la ciudad. El nuevo Madrid de la Feria del Libro, inaugurada en 1933, del Instituto-Escuela y la investigación abierta a Europa comenzaba a identificarse con la metrópolis (Holguín, 2003; Martínez Rus, 2003; Otero Carvajal y López Sánchez, 2012; Laporta San Miguel et al., 1987; Martínez Alfaro, 2009).

Sin embargo, la separación y afirmación autónoma de los mundos público y privado, la irrupción de las "masas" en la vida política y la aparición sostenida de la mujer en el espacio público, consecuencias de la Modernidad, no formaron parte de un proceso lineal ni carente de resistencias. Fue entendido por diversos sectores como el socavamiento de los principios tradicionales. Al mismo tiempo, la ciudad también fue escenario de la aparición de nuevas formas de conflictividad, que giraron del motín de subsistencias hacia la huelga (Otero Carvajal, 2007; González Calleja, 1998; Sánchez Pérez, 2005). Fue, en cierta forma, una cuestión de orden público ${ }^{2}$ que se proyectaba desde finales del siglo XIX y que tuvo su marco de desenvolvimiento en el primer tercio del siglo Xx. Las élites del sistema de la Restauración reaccionaron ante los nuevos repertorios de acción colectiva y las nuevas formas de comportamiento, que parecían desbordar los límites de su arquitectura política. En este sentido, el mantenimiento del orden público se equiparó a la defensa del orden social, entendido como "plasmación inamovible, naturalizada e idealizada de los valores e intereses de los grupos dominantes" (González Calleja, 2008: 94), lo que animó a reforzar la presencia del Ejército en los aparatos de coerción, a la suspensión de las garantías constitucionales y la aplicación multiplicada de medidas de excepción. El aumento de las distintas formas de represión indicaba, como apuntó Rafael Cruz, una crisis del Estado, que se extendió a los años republicanos, puesto que también hubo descoordinación entre agencias y se aprobaron algunas medidas legales de excepción. Quedaba claro que, en materia de orden público, el régimen del 14 de abril tuvo que hacer frente a dos grandes problemas: no depuró el aparato coactivo y utilizó de forma inadecuada los diferentes cuerpos de policía, tanto en la ciudad como en el campo (González Calleja, 1999; Cruz, 1993; González Calleja, 2014).

En el mundo urbano, el miedo a que la protesta se adueñara del centro histórico se instaló en amplias capas de la población y la representación del delito pasó del temor a la diferencia, a lo desconocido, propio del siglo XIX, a convertirse en miedo al adversario político a la par que la ciudad crecía y se descubrían nuevos espacios. La identificación de las clases populares con unas clases peligrosas y del conflicto con el delito articuló un discurso conservador y reaccionario que sentía que la ciudad había dejado de pertenecerles (Gómez Bravo y Pallol Trigueros, 
2013; Vicente, 2014). Pensar este proceso como una oposición mecanicista entre Modernidad y Tradición (o reacción) equivale a desdibujarlo, a trazar una línea sin solución de continuidad entre la República y la guerra. La conflictiva relación entre esa multiplicidad de expresiones socioculturales, entre formas variadas de concebir las relaciones (sociales, laborales, de género) y el ocio, las nuevas expectativas y deseos ante el futuro fue el caldo de cultivo para quienes teorizaron la necesidad de un golpe de Estado que recondujera la situación, no la causa inexorable de la guerra. En julio de 1936, la experiencia republicana y el desarrollo de su marco legal, en el que se podían dirimir esos conflictos, fueron abortados por una sublevación militar que entendió la violencia como el recurso más adecuado para modelar la sociedad deseada.

\section{LA GUERRA COMO MARCO DE REACCIÓN}

Todos nuestros males vienen de las alcantarillas. Las masas de este país no son como sus americanos, ni como los ingleses. Son esclavos. No sirven para nada salvo para hacer de esclavos. Pero nosotros, las personas decentes, cometimos el error de darles casas nuevas en las ciudades en donde tenemos nuestras fábricas. En esas ciudades construimos alcantarillas, y las hicimos llegar hasta los barrios obreros. No contentos con la obra de Dios, hemos interferido en Su Voluntad ${ }^{3}$.

Así explicaba a finales de 1936 el capitán Gonzalo de Aguilera, conde de Alba de Yeltes, los motivos por los que se combatía en campo franquista a Charles Foltz, corresponsal de Associated Press en España. La sublevación apuntaba a la ciudad como el lugar donde la cosmovisión tradicional había colisionado con el ascenso de las masas en el espacio público. Los años republicanos representaron la colmatación de toda una serie de demandas sociales: la extensión del sufragio a sectores hasta entonces marginados, la tensión en el régimen de propiedad, la laicidad del espacio público y por tanto del Estado o una legislación que diera soporte jurídico a las nuevas condiciones sociales y económicas. Las palabras de Aguilera no eran ingenuas y el momento, clave. El Ejército franquista, que había avanzado desde África hasta las puertas de la capital en menos de cuatro meses, acababa de dar por fracasado el asalto frontal a la ciudad. En noviembre de 1936, ante Madrid, cambió la forma de hacer la guerra. La marcha del conflicto hizo que Franco y su Estado Mayor fueran conscientes de las nuevas exigencias. A ello dedicaron los primeros meses de 1937: inserción de las milicias en las estructuras militares mediante las escuelas de alféreces provisionales, mejor aprovechamiento del material extranjero en las operaciones o la formación de un ejército de mayores dimensiones a través de las reclutas, fueron solo algunas de las medidas adoptadas (Aróstegui, 1996).

La principal tarea fue el paulatino desarrollo del espionaje, lo que ya indica que en el Cuartel General de Franco los altos mandos estaban reflexionando sobre la importancia de preparar más detenidamente las operacio- nes militares. En la primera mitad de 1937 entró en escena uno de los personajes fundamentales para entender la forma de operar de los servicios de información franquistas, y por tanto, para entender los planes de ocupación del territorio republicano. Su nombre era José Ungría, un militar destinado en el Estado Mayor de la División de Caballería de Madrid, refugiado desde verano en la Embajada francesa. Había logrado salir de la capital, donde consiguió levantar una red de espionaje y otra de apoyo en colaboración con falangistas y miembros de la incipiente Quinta Columna, que, según Javier Cervera, empezaba a dar sus primeros pasos (Cervera, 1998: 260). En mayo de 1937 se hizo con las riendas del Servicio de Información Militar tras denunciar el excesivo número de servicios existentes y su escasa coordinación. Sin duda alguna los conocimientos adquiridos en la Escuela Superior de Guerra de Madrid y la École Superieur de Guerre de París durante los años 20 fueron de gran ayuda para convencer a sus compañeros de armas de la necesidad de implementar técnicas propias de una guerra moderna (Heiberg y Ros Agudo, 2006; Bahamonde y Cervera, 2000: 264).

En paralelo, en la primavera de ese mismo años se formaron otros organismos. Quizá el más importante de ellos fuera la OIPA, la Oficina de Investigación y Propaganda Anticomunista, dirigida por Marcelino de Ulibarri, jefe de la Junta Central Carlista de Guerra en la merindad de Tudela y un carlista muy próximo al conde de Rodezno. Junto a Ungría, hay que considerar a Ulibarri una de las figuras cruciales, y más desconocidas, en la progresiva estructuración del nuevo Estado franquista y la superación de las imprecisas funciones propias del "Estado campamental", pues ya había formado parte de la Oficina de Prensa y Propaganda en Pamplona al comienzo de la guerra. Dependiente de la Junta, esta oficina jugó un papel fundamental tanto en la difusión del ideario tradicionalista como en la recogida de las informaciones que en la prensa extranjera aparecían sobre la guerra de España. Seguía también la actividad de la zona republicana a través de sus diarios, para así conocer el estado de opinión en el campo enemigo, las operaciones militares de las que se hacía eco la prensa y la reacción ante las ofensivas franquistas (Ollaquindía, 1995). La OIPA se creó el 20 de abril de 1937 como una agencia de contrainformación dependiente de la Secretaría General del jefe del Estado, con la misión de

recoger, tanto en la Zona ocupada como en las que se vayan ocupando, la mayor cantidad de pruebas de las actividades marxistas en España [...]. Para el desempeño de su importante misión, los miembros de la Oficina de Investigación y Propaganda anti-Comunista necesitarán de la ayuda y cooperación de las Autoridades Militares y Civiles, por lo que se ruega a las mismas les presten el más decidido apoyo ${ }^{4}$.

Para ello, se promovió la colaboración con instituciones europeas tales como el Antikomintern de Berlín, el Instituto de Investigación Científica del Comunismo de 
Varsovia, la institución anti-marxista "Maitre Aubert" de Ginebra o la "National Herstel" de Holanda. Los masones, el Socorro Rojo Internacional, las ligas antifascistas o de Derechos del Hombre, los ateneos libertarios, la Federación de Trabajadores de la Enseñanza de la UGT o las instituciones naturistas eran algunos de los colectivos que estaban en el punto de mira de la OIPA. Amigo personal de Franco, secretario y consejero, Ulibarri estaba en condiciones inmejorables para conocer al enemigo a través de sus documentos y poder contrarrestar su maquinaria propagandística, así como influir en la forma de combatirlo encauzando su represión y control (Gómez Bravo y Marco, 2011: 160).

A la altura del ecuador de la guerra y en paralelo a esta reglamentación, los diferentes servicios de inteligencia franquistas seguían con los ojos puestos en la capital. La progresiva mejora en el conocimiento de lo que pasaba en su interior, junto a la experiencia acumulada en la conquista de diversas ciudades del Norte, dio como resultado nuevas reflexiones sobre la futura ocupación del resto de grandes núcleos urbanos. En algunos casos, reflexiones nada complacientes, como la que sigue:

En el caso de una caída vertical de unos de estos frentes tenemos el ejemplo de Gijón, al ocuparse ciudades de la importancia de Madrid, Valencia y Barcelona, incluyendo las correspondientes a estas regiones de menor importancia como son Gerona, Lérida, Castellón de la Plana, etc, es una cosa harto segura que el Servicio fracasaría por falta de elementos. Y precisamente fracasaría en aquellos centros donde los enemigos han actuado con mayor intensidad y más cantidad de elementos extranjeros rusos y de las brigadas internacionales ${ }^{5}$.

La experiencia de la súbita caída de Gijón, el 21 de octubre de 1937, demostró que no bastaba con ocupar una ciudad, había que anticipar, en la medida de lo posible, cómo iba a suceder. La labor de Ulibarri no se detuvo y antes de finales de año terminó de perfilar el diseño del servicio de recuperación de documentos a través de dos textos internos cruciales: el propio reglamento y las normas para la entrada en una ciudad ocupada. El primero era un estudio dividido en cuatro capítulos, desde la formación de los propios equipos a la dotación económica del servicio, pasando por el análisis de la clasificación y la creación de una oficina general. Cada equipo de recuperación estaría formado por un jefe, dos auxiliares y un conductor, un equipo que a través de su jefe estaría en contacto diario con el jefe del servicio de Recuperación, al que habría de enviar un informe semanal sobre el curso de las actividades. Las oficinas de clasificación eran los núcleos locales del servicio, centralizando la labor de los equipos y realizando una primera labor de clasificación, que habrían de remitir a la Oficina Central. Estas oficinas de primera clasificación estarían conformadas por un jefe de servicio, un equipo de clasificación y los equipos de recuperación que se les asignaran, instalados en un local de fácil comunicación para asegurar una rápida y flexible coordinación. Los equipos de clasificación eran los encar- gados de recoger la documentación de los jefes de equipo, que debían encargarse personalmente de entregársela en mano. Es decir, desde la unidad básica del servicio, el equipo, a la oficina central de clasificación, se puede apreciar que Ulibarri buscaba tanto jerarquía como flexibilidad para asegurar un trabajo bien hecho. Era la primera vez que en el campo franquista se recogía información del enemigo de forma sistemática, con el objetivo de clasificarla y así encauzar la represión de las zonas ocupadas. Por tanto

La clasificación ha de hacerse someramente, teniendo en cuenta la procedencia y pensando en el Organismo a que puedan ser más útiles: Auditoría, Policía, Departamento del Gobierno, Asuntos Militares, de Estado Mayor, etc... Cualquier duda debe consultarse por el que la tuviere al Jefe de Clasificación, quien despachará con el Jefe del Servicio y tendrá en definitiva la responsabilidad de la actuación del grupo de clasificación ${ }^{6}$.

El celo por cuidar la cadena de mando queda fuera de toda duda. Sólo la disciplina era aval del éxito del servicio de recuperación, que debía entrar (esto era crucial) junto con las tropas de ocupación. "El trabajo que no se realiza dentro de los cuatro primeros días, deja de tener eficacia", rezaba el primer párrafo de las "Normas para la entrada en una ciudad ocupada". Como la experiencia había demostrado que era imposible registrar todos los locales en un plazo de tiempo tan exiguo, la primera y principal labor de los equipos debía ser la de precintar los domicilios que previamente habían elegido ${ }^{7}$. Ante tal volumen de posibles registros y con edificios tan diversos, había que extremar el cuidado, aunque quedaban claros los objetivos principales a la hora de la ocupación de los núcleos: la búsqueda de responsabilidades, como ya expresara Franco meses atrás.

Quedaba muy claro que el perfeccionamiento del modelo de ocupación era producto de la coordinación de múltiples agentes, entre los que habría que destacar por encima de todas las figuras de Ungría y Ulibarri. Probablemente no sea una exageración decir que el año de 1937 marcó un verdadero antes y después en el conocimiento del campo republicano y, por tanto, en el tipo de guerra que se pretendía llevar a cabo. Y todo ello se hizo a través de gestiones entre las bambalinas del poder militar, como ya avanzaron Gutmaro Gómez Bravo y Jorge Marco:

Entre los meses de mayo de 1937 y julio de 1938 se emitieron una serie de normas y decretos reservados, que establecieron la conexión entre los distintos servicios sobre los que recaerá el control del orden público de ahora en adelante: la ocupación y la justicia militar, el control policial a través de la Policía Política, precedente de la Dirección General de Seguridad, y la investigación de los antecedentes político-sociales de cada individuo a través del Servicio de Recuperación de Documentos y su conexión con la posterior Brigada Político-Social (Gómez Bravo y Marco, 2011: 159160). 
Precisamente ése era el contexto en que se inscribe toda esta labor normativa, hasta ahora casi desconocida. El esfuerzo en la sistematización de la información capturada al enemigo tras la ocupación de su territorio estaba claramente orientado al castigo de la movilización política en los años anteriores a la guerra y a la prevención de lo que se equiparaba al desorden. La ocupación del territorio y el control del mismo eran dos etapas unidas por la misma secuencia, en la que la guerra prepararía los ejes de la sociedad de posguerra. Se confiaba de nuevo en las labores de policía, pero fiadas a un conjunto de personas que hubiesen pasado por un organismo determinado: el Ejército. La definición del franquismo como un régimen preeminentemente de control, aún en un estado embrionario, aparecía ya en plena guerra, unido a la firme preocupación por manejar información del enemigo. Una constante, por otro lado, en todas las guerras civiles (Kalyvas, 2006: 146-209) y que en España ya se había practicado, a efectos punitivos, en la ocupación de Málaga (Anderson, 2008).

Justicia militar, policía política, Dirección General de Seguridad, Brigada Político-Social... Diversos organismos que deberían ser armonizados para su buen funcionamiento. La coordinación era el punto en que ahondaba otro documento reservado, titulado simplemente "Recuperación de Documentos", un nuevo organismo que aprovechó la experiencia de la OIPA y amplió sus funciones en relación al enemigo. Tras una breve explicación de su función, Ulibarri pasaba a explicar la relación con otras instancias oficiales, que expresaba de dos maneras: primero como elemento auxiliar, es decir, para proporcionar todos aquellos datos que pudieran ser de interés a todos los organismos del nuevo Estado; y segundo, para aprovecharse de ellos en relación con la función privativa, por ejemplo la policía. Sin embargo, el mayor interés estaba puesto en la coordinación con la Auditoría del Ejército de Ocupación, la principal agencia que iba a impartir justicia en los territorios "liberados": "como quiera que la citada Auditoría prepara su sección de información con vistas a actuaciones futuras, es útil el servicio de recuperación ensanchando sus medios de adquisición de datos interesantes 8 ".

Había llegado el momento de preparar la ocupación de las grandes ciudades que todavía permanecían en poder de la República. En el caso particular de Madrid, los dos últimos meses de 1937 son un buen ejemplo de la complejidad de la ocupación que proyectaban. Fue un momento de máxima complicidad entre la labor reglamentista de Ulibarri y la presión de Ungría por reorganizar los servicios de inteligencia. Como ya expusieron Martin y Joan Kyre, en las guerras de liberación es fundamental la colaboración de agencias civiles y militares para las tareas de ocupación, "pacificación" y gestión de la sociedad post-conflicto (Kyre y Kyre, 1968: 68-73 y 99-122). En el caso español ocurría algo similar. Recuperación de Documentos, dependiente del Ministerio de Gobernación pero coordinada por un militar (Ulibarri), y su constante comunicación con la Auditoría y el SIPM era un buen ejemplo de esa necesidad.
Había que centralizar la labor de los diferentes organismos y otorgar al espionaje una dimensión política y militar. Para coordinarse mejor con el Cuartel General de Franco, la oficina debía trasladarse a Burgos. Después de los intentos de Ungría por convencerle durante el verano, el Caudillo aceptó en octubre su propuesta y le dio instrucciones para promulgar toda una serie de medidas secretas que culminaron en la creación, el 30 de noviembre de 1937, del Servicio de Información y Policía Militar (SIPM). Entre los 17 artículos de la orden de creación, destacan por encima del resto los que llamaban la atención sobre la necesidad de homogeneizar todas las secciones de contrainteligencia, de mantener separadas las funciones de los servicios secretos respecto de las que llevaban a cabo las grandes unidades militares y de proveer de mejores medios económicos y humanos el servicio "para la consecución de los objetivos de sus funciones especiales"

Este último punto coincidía plenamente con las preocupaciones de Ulibarri, como delegado de Recuperación de Documentos, sobre la capacidad efectiva de actuación de su servicio. Además, para asegurar el dominio sobre el territorio enemigo, dentro del SIPM existía un Servicio de Vigilancia, Seguridad y Orden Público, que debía controlar la zona de frente en unos 30 kilómetros de profundidad más allá de la línea de combate. El propio servicio creó sus secciones adjuntas al cuartel general de cada gran unidad militar, tipo división, y las fuerzas de la Policía Militar de cada sector dependían del oficial del SIPM asignado a cada unidad del Ejército (Heiberg y Ros Agudo, 2006: 93-96). Es decir, que ocupación y orden público aparecían más estrechamente unidas que nunca desde sus primeras teorizaciones, lo que se reafirmaba a través de la responsabilidad exclusiva del SIPM de la retaguardia del enemigo.

¿Cómo afectó este proceso a la planificación de la ocupación de la capital? A la altura de noviembre de 1937, la delegación de Madrid del Servicio de Recuperación de Documentos estaba ya preparada para actuar. Es decir, en la mente de los militares, la ocupación de Madrid era un horizonte a cumplir, si no ese año, sí para el siguiente. Con siete equipos de clasificación, parece que las presiones de Ulibarri por aumentar los efectivos dieron sus frutos. La delegación estaba compuesta por cinco secciones, con sus responsables nombrados, más el jefe de clasificación y el de servicios, responsable del conjunto. $Y$ hay que añadir una cuestión importante: el servicio se denominaba "Delegación de Servicios Especiales y Recuperación de Documentos ${ }^{10}$ ". La requisa de material al enemigo cumplía una función mayor, como muestra la Tabla 1.

La delegación de Madrid se presenta así como un ámbito privilegiado para observar la plasmación de las preocupaciones de Ulibarri en el funcionamiento práctico. En primer lugar, el jefe de los servicios era un auditor militar, por lo que la recuperación de documentos se encaminaba definitivamente a la búsqueda de antecedentes y la impartición de justicia en los territorios "liberados". Los siete equipos de recuperación, aquellos grupos de operación 
TABLA 1. Servicios Especiales y Recuperación de Documentos. Delegación de Madrid.

\begin{tabular}{|c|c|c|}
\hline Nombre & Cargo & Empleo militar \\
\hline Javier Dusmet & Jefe de Servicios & Coronel auditor \\
\hline Sr. Íñiguez & Jefe de Clasificación & \\
\hline Sr. González de Andía & Jefe $1^{\text {a }}$ Sección: Político-Social & \\
\hline Sr. Carrillo de Albornoz & Jefe $2^{\text {a }}$ Sección: Prensa y Propaganda & Alferez castrense \\
\hline José Gómez & Jefe $3^{\text {a }}$ Sección: Sectas secretas & Capitán de la Guardia Civil \\
\hline Jesús Muñoz & Jefe $4^{\text {a }}$ Sección: Justicia & Alférez del Cuerpo Jurídico \\
\hline Manuel Sanchíz & Jefe $5^{\text {a }}$ Sección: Militar & \\
\hline
\end{tabular}

Fuente: CDMH, DNSD, Recuperación, Secretaría, Delegación de Madrid, Caja 11, Expediente 7. Elaboración propia.

que iban a iniciar el contacto directo con el material, se lo transferían directamente al jefe de clasificación, que lo repartía en cinco secciones independientes: Político-Social, Prensa y Propaganda, Sectas secretas, Justicia y Militar. De las cinco, tres se destinaban a la creación de un fichero de antecedentes políticos, otra se encaminaba a la puesta en funcionamiento de la justicia de ocupación y tan sólo la última estaba en consonancia con las operaciones militares. Quedaba así plasmada la colaboración Ulibarri-Ungría, pues la delegación estaba volcada en la recuperación de material político-social. Además, de siete jefes, tres estaban relacionados con la justicia militar y otro, el jefe de la Tercera Sección, pertenecía a un cuerpo experto en control, la Guardia Civil.

Tras año y medio de guerra, en la zona franquista las reflexiones sobre la violencia tendían más hacia el perfeccionamiento de las herramientas de control en la ocupación de grandes ciudades que hacia el exterminio del enemigo. Desde este punto de vista, la violencia tuvo un carácter eminentemente funcional, de parálisis social a través de la eliminación y el castigo. Mientras la primera se encauzaba a través del frente y los asesinatos extrajudiciales, el castigo se imponía preferentemente a través de la maquinaria de los consejos de guerra, que tomaron protagonismo desde febrero de 1937, con la caída de Málaga (Prada, 2010: 61-69 y 163-215). A medida que avanzaba el conflicto, en el Estado Mayor del Generalísimo se impuso la necesidad de pensar cómo se iba a desarrollar la ocupación de los grandes núcleos de población, pues ése era su objetivo con la guerra decantada a su favor. A principios de diciembre, Franco decidió que su próximo ataque iba a darse en Madrid, en una ofensiva en dirección a Alcalá de Henares para completar el cerco que los italianos no pudieron llevar a cabo en marzo durante la batalla de Guadalajara. De ahí que se hubiera acelerado el afinamiento de las labores de recuperación de documentos y de los servicios de orden. Sin embargo, otra vez el contexto imposibilitaba completar sus planes: el general Rojo lanzó una ofensiva el 15 de diciembre en Teruel y Franco tuvo que abandonar su objetivo (Puell de la Villa, 2012: 207-220; Martínez Bande, 1980: 16-26).

\section{MILITARIZAR LA CIUDAD, CONTROLAR LOS BARRIOS: LA PLASMACIÓN EN EL ESPACIO URBANO}

La organización de la recuperación de documentos estaba ultimada pero, ¿qué ocurrió con el orden público? A mediados del mes de diciembre de 1937 se elaboró el "Plan de Orden y Policía para Madrid, Barcelona y Valencia" ${ }^{11 "}$, donde las tres capitales de la República recibían el mismo trato, aunque estaba firmado por los Servicios de Orden y Policía de Madrid (Ejército del Centro). Es un documento importante porque adelanta ocho meses la fecha que se conocía hasta ahora para esta planificación (Gómez Bravo y Marco, 2011: 165-169). La principal consigna del plan era clara: nadie podía salir o entrar de la ciudad ocupada a menos que dispusiera de un salvoconducto expedido por Franco, el General Jefe del Ejército de Ocupación o el Jefe de la Columna de Orden y Policía. Es decir, se pretendía convertir la ciudad en un espacio cerrado y prácticamente inaccesible, de cara a facilitar la labor de la Auditoría de Guerra y del Servicio de Recuperación, ya que el objetivo principal era la elaboración de un censo provisional de la ciudad y castigar a aquellas personas con responsabilidades políticas y militares. Para ello, todos los ocupantes de pisos, habitaciones y locales estaban obligados a presentarse en las comisarías de distrito y ofrecer una relación jurada de los habitantes, en un plazo máximo de 24 horas. Dentro del mismo plazo había que ofrecer otra relación, esta vez con los bienes muebles existentes en cada casa. Los militares también controlarían el movimiento dentro de la ciudad mediante cédulas de transeúnte y reforzarían las labores de vigilancia con cédulas de Policía, de las que se proveería únicamente a los residentes en la ciudad con anterioridad al 18 de julio de 1936. De esta forma se reforzaba el celo sobre los atentados contra la propiedad acudiendo a los espacios mínimos de sociabilidad: los barrios y las casas.

En el tránsito de 1937 a 1938 se aprecia un trasvase de funciones desde Recuperación de Documentos a la Columna de Orden, es decir, desde la ocupación al control efectivo. O, en otros términos, desde la voluntad de obtener información a la preocupación por el diseño de un or- 
den público eficiente. De hecho, los Servicios Especiales ya aparecían incrustados en un esquema puramente de orden público, guiado exclusivamente por militares. Era lógico, puesto que iban a ser éstos los primeros en entrar en los grandes núcleos de población aún en poder de la República. En el tramo final de la guerra, Franco se decantaba por potenciar su modelo de orden, frente a un servicio que había tenido errores en sus primeras misiones durante la campaña del Norte. Por si no era suficiente, los jefes de sector también estaban implicados en el mantenimiento del orden público y el desarrollo de políticas activas de control en el espacio, pues eran responsables de los nombramientos de los jefes de barrio y casa:

Dependientes del Jefe de Sector, se nombrará en cada barrio una persona de reconocida solvencia a la cual quedarán subordinados los Jefes de casa (uno debidamente garantizado por cada edificio) siendo la única misión de unos y otros suministrar datos para confeccionar con toda rapidez el fichero policíaco de la Capital ${ }^{12}$.

Es decir, la estructura de control dominante en la posguerra, que ha quedado grabada en la memoria colectiva a través de las novelas (Cela, 1951; Martín-Santos, 1961) y calificada como "falangista" (Montoliú, 2005: 47; García Ballesteros y Revilla González, 2006: 7), era realmente un diseño puramente militar, organizado jerárquicamente. Para ello contaba con individuos que se hubiesen destacado por su identificación con los valores del Movimiento en el Madrid ocupado por los "rojos". Esto les reportaría ascendencia sobre las comunidades de vecinos y la capacidad de informar sobre sus antecedentes, puesto que su objetivo era el de colaborar con las autoridades en la elaboración de un fichero policial. La militarización de la calle sería la forma de reproducir el orden social que, según el imaginario sublevado, había estado a punto de desaparecer. Se recurría a colaboradores de diferentes procedencias, sí, pero en la cúspide de la pirámide se situaba el Ejército, encargado de ser el factor (amenazante) que salvaguardara unas relaciones de dominación asentadas en amplios sectores de la población. Como en otras ocasiones a lo largo de la historia, la coacción se convertía en un elemento fundamental en la reproducción de un orden social concreto (Duménil y Lévy, 2011).

Por otro lado, y de cara a evitar fugas, en las carreteras de entrada y salida se establecerían cordones de patrullas que exigirían autorizaciones firmadas por el propio Generalísimo o el Ministro de Orden Público para el tránsito, incluso a grupos de soldados que no estuvieran liderados por su jefe y marcharan formando una unidad organizada. La lucha contra el anonimato, un fenómeno propio del mundo urbano, suponía desarrollar un conjunto de prácticas bastante alejadas del modelo de orden público establecido en el campo, donde la aparición de las columnas dio paso a las gestoras municipales y a una represión liderada por las milicias (Cobo Romero, 2005; Gil Andrés 2006; Melero Vargas, 2013). La limpieza política del pueblo tenía como efecto la paralización social a través del miedo (Marco, 2011: 71-83 y 293-318). El con- trol de una gran ciudad como Madrid implicaba la creación de instancias más complejas (Gómez Bravo y Marco, 2011: 179-200), como ilustra la Tabla 2 para la organización de una Jefatura de Sector.

TABLa 2. Composición de una Jefatura de Sector.

\begin{tabular}{|l|}
\hline 1 Jefe militar (Teniente Coronel, Comandante o Capitán). \\
\hline 1 Inspector Segundo, Jefe del Cuerpo de Investigación y \\
Vigilancia. \\
\hline Personal civil de oficinas \\
\hline 10 Agentes del Cuerpo de Investigación y Vigilancia \\
\hline 1 Compañía de la Guardia Civil, al mando de sus Oficiales \\
\hline 40 individuos de Milicias, de ellos diez especializados \\
\hline Un Batallón de Orden Público \\
\hline
\end{tabular}

Fuente: AGMAV, Caja 2552, Carpeta 44. Elaboración propia.

Más adelante, las bases incidían en la organización de las Jefaturas de Sector, que habrían de dividirse en Negociados y Secciones para el despacho de asuntos:

\begin{abstract}
En aquellas poblaciones que por su importancia merezcan especial atención y muy particularmente en las de Madrid, Barcelona y Valencia, se procurará dividirlas en tantos Distritos como, para efectos de Censo, estuvieran ya divididas con antelación, encargándose de cada Distrito un Jefe. Para facilitar su labor al tiempo de hacerse cargo de su cometido, convendrá realizar con anterioridad un trabajo preparatorio con planos dividiendo las poblaciones en sus Distritos respectivos, y estos en barrios o zonas, para que llegado el momento, cada Jefe de Sector pueda conocer perfectamente la parte que le corresponde. [...] Se realizarán de antemano cuantos trabajos puedan redundar en beneficio de la mejor organización y más fácil conocimiento de los respectivos distritos y de las personas que en ellos hubieran residido, tomándose los antecedentes precisos relacionados con los servicios de orden y policía (detenciones, protecciones, etc. etc.) a fin de que inmediatamente de tomar una población se pueda comenzar a actuar eficazmente y sin pérdida de tiempo ${ }^{13}$.
\end{abstract}

Los sectores en que quedaban divididas las ciudades coincidían con los distritos, las divisiones administrativas tradicionales, para facilitar las labores de control y coordinación. Este trabajo debía hacerse previamente a la ocupación, por lo que es de suponer el contacto con los elementos de la Quinta Columna para el conocimiento de los barrios "a ras de suelo". En plena guerra las autoridades confiaron en el sector, dirigido por un militar, como eje espacial del control social en la ciudad. Éstos pasarían a ser grandes núcleos militarizados a efectos de diseño e iniciativa, en los cuales los jefes de sector eran militares y a su cargo tenían un batallón de orden público, como muestra la Tabla 3. Este paisaje urbano militarizado ${ }^{14}$ era la expresión espacial del modelo de orden público pensado entre 1937 y 1938 por las autoridades franquistas. 
Desde el exterior de la ciudad, con los controles de carretera, al interior de la misma, con las comisarías de distrito ocupadas por oficiales y batallones de orden público, la capital quedaría completamente en manos del Ejército, replegado hacia la vigilancia interior, lo que inauguraba el patrón de orden vigente durante toda la dictadura. Un control que se proyectaba hacia los mismos barrios, donde residían las pautas mínimas de sociabilidad en la gran ciudad. La presencia civil en la orientación de las divisiones básicas de la capital quedaba apartada en favor de la primacía castrense, al frente de los distritos.

Todo estaba preparado para lanzarse al asalto y control de la capital. Todos los distritos tenían un batallón de orden público asignado, salvo Buenavista, donde antes de la guerra residían los apoyos "naturales" al orden social tradicional. Quizá en la mentalidad de los ocupantes éste fuera un espacio fácil de controlar, o quizá no había suficientes fuerzas para formar diez batallones completos. Lo cierto es que si bien ya estaba preparada la estructura de dominio de la ciudad, también lo estaban los servicios para gestionar la urbe en los primeros momentos tras la ocupación, con los nombramientos de sus responsables:

Madrid sería administrada única y exclusivamente por militares, encargados de hacerse con los resortes de una metrópolis moderna. Destaca la importancia otorgada a la gestión de los organismos responsables de la vida cotidiana: transportes, abastecimiento y sanidad, fundamentalmente. El SIPM conocía bastante fielmente las condiciones de vida de Madrid y a través de éste, Ungría y el propio Franco. Sobre todo, cuando era la propia Quinta Columna la que colaboraba activamente en el sabotaje cotidiano, la extensión del derrotismo y el empeoramiento de las condiciones de habitación en una ciudad asediada (Cervera, 1998: 199-212). Era lógico potenciar también este aspecto de la ocupación. Las propias autoridades civiles (Ayuntamiento, Diputación...) eran nombradas por los militares. Por no hablar de cuerpos ya considerados clave, como Justicia, Correos y Telégrafos o Abaste- cimientos, donde la primacía castrense es incuestionable. La Tabla 4 revela en todas sus dimensiones la concepción de una ciudad militarizada.

La persistencia de esta consideración en los planes de ocupación de las grandes ciudades, con un desarrollo muy perfeccionado para Madrid, hace pensar en una obsesión de Franco por entrar en la que había sido capital de la resistencia republicana. ¿Su caída habría supuesto el final de la guerra, un año o dos antes de que finalmente llegara a suceder? ¿La conquista de Madrid habría significado la conclusión de la resistencia republicana? Por encima de una reflexión imposible de documentar hay que destacar la primacía del Cuartel General del Generalísimo en el desarrollo de una estructura de control adaptada a las ciudades. Los mismos espacios urbanos de los que los poderes tradicionales sentían haber perdido el control. Por tanto, es necesario diferenciar entre el conjunto de agencias y aparatos relacionados con el orden público, completamente modernos, y el propio orden público que se pretendía imponer, expresión del rechazo a la Modernidad característica del mundo urbano. Una interpretación que matiza la canónica sobre el franquismo, que insiste en que "el llamado «nuevo Estado» no es tal si nos ceñimos al conjunto de aparatos y técnicas jurídicas relacionadas con el orden público" (Ballbé, 1985: 399).

La tesis de la continuidad no tiene en cuenta la profunda fractura que supuso la guerra, entendida como el marco en que se concretó la reacción contra una serie de procesos a los que el franquismo dio respuesta en forma de una estructura de control bastante colmatada. Antes de la última gran batalla de la guerra, la del Ebro, esa estructura quedaba proyectada sobre la vigilancia de los distritos y la gestión cotidiana de Madrid. En el Servicio de Orden y Policía de Madrid estaban insertos tanto el Cuerpo de Investigación y Vigilancia como el Servicio Especial de Recuperación de Documentos, a cargo de un coronel perteneciente a la Auditoría de Guerra, con la finalidad de impartir justicia en el territorio ocupado. La coordina-

Tabla 3. Jefes de Batallón de Orden Público. Columna de Orden y Policía de Madrid. Abril de 1938.

\begin{tabular}{|l|l|l|l|}
\hline \multicolumn{1}{|c|}{ Nombre } & \multicolumn{1}{c|}{ Empleo militar } & \multicolumn{1}{c|}{ Batallón asignado } & \multicolumn{1}{c|}{ Distrito } \\
\hline José María Cabanillas Prosper & Comandante & 402 & Centro (1) \\
\hline Manuel Losada Roces & Comandante & 405 & Hospicio (2) \\
\hline Julio Pérez Pérez & Comandante (Guardia Civil) & 417 & Chamberí (3) \\
\hline Enrique González Conde de Illana & Teniente Coronel & El que se designe & Buenavista (4) \\
\hline Julio Condo & Comandante & 418 & Congreso (5) \\
\hline Luis Serrano Marenjes & Teniente Coronel & 416 & Hospital (6) \\
\hline Manuel Sánchez Molina y Mendoza & Comandante & 406 & Inclusa (7) \\
\hline Alfonso Morandeira & Comandante & 401 & Latina (8) \\
\hline José Luque de Riocanal & Comandante & 421 & Palacio (9) \\
\hline Isabelo Aguado Martínez & Comandante & 424 & Universidad (10) \\
\hline
\end{tabular}

Fuente: AGMAV, Caja 2552, Carpeta 43; AGMAV, Caja 2552, Carpeta 44. Elaboración propia. 
Tabla 4. Jefes de los distintos servicios de Madrid. Columna de Orden y Policía de Ocupación. Abril de 1938.

\begin{tabular}{|l|l|}
\hline \multicolumn{1}{|c|}{ Nombre } & \multicolumn{1}{c|}{ Servicio } \\
\hline Juan de Villalonga & Abastecimientos \\
\hline Juan Petrirena ${ }^{15}$ & Aguas \\
\hline Alberto de Alcocer & Servicios urbanos, bancarios y de índole varia \\
\hline Javier Martínez de Bedoya & Beneficencia \\
\hline José Luis Anchústegui Nardiz & Cámara de Comercio e Industria \\
\hline José Tristán Palacios & Carabineros \\
\hline Santiago Noreña Echavarria & Correos y Telégrafos \\
\hline Manuel Antonio García Alegre & D.I.D.R.E.M. ${ }^{16}$ \\
\hline Samuel Crespo & Diputación Provincial \\
\hline Francisco Lezcano & Electricidad \\
\hline Víctor Mesa Aznar & Ferrocarriles \\
\hline María Rosa Urraca Pastor & Frentes y Hospitales \\
\hline José María Rezola & Gas \\
\hline Pedro Simarro Roig & Guardia Civil \\
\hline Ángel Manzaneque & Justicia \\
\hline Carlos Laffite & Metropolitano \\
\hline Julio Pérez y Pérez & Milicias FET y de las JONS \\
\hline José María Echevarría & Radio \\
\hline Carlos Velasco & Prisiones \\
\hline José Alberto Palanca & Sanidad \\
\hline Manuel Martínez Franco & Teléfonos \\
\hline José Luis Escario & Trabajo \\
\hline Augusto Krahe & Tranvías \\
\hline Fernando Vélez y Gumersindo García & Falange \\
\hline & \\
\hline
\end{tabular}

Fuente: AGMAV, Caja 2552, Carpeta 44. Elaboración propia.

ción de tres agencias fundamentales para comprender la posguerra representaba al mismo tiempo la preparación de la entrada en la capital y el perfeccionamiento de los métodos represivos del régimen.

\section{REFLEXIONES FINALES: HACIA UNA REINTERPRETACIÓN DE LA GUERRA Y EL FRANQUISMO}

Sabe esperar días y meses a las puertas sin hojas ni goznes del claro Madrid, del Madrid frágil, sedoso y fastuoso; del Madrid en colapso fantasmal indecible, del Madrid de ayer, rescoldo rubicundo soterrado [...]

La obra de Franco no es material: va por las almas y la Historia. Todo está previsto por Franco y por él proyec$\operatorname{tado}^{17}$.
En el mes de abril de 1938, cuando Franco consiguió seccionar el territorio republicano en dos y aislar Cataluña de Madrid, la ocupación de las grandes ciudades estaba ultimada. En los planes del alto mando militar estaban presentes las tres capitales de la II República en guerra, pero Madrid destacó sobre el resto por su planificación autónoma y detallada. Entre el fracaso de su asalto directo en diciembre de 1936 y el inicio de la batalla del Ebro, es decir, el momento central de la guerra, la preparación de la entrada en Madrid motivó en la zona franquista una serie de reflexiones sobre el orden público que se quería instaurar en las zonas ocupadas.

A medida que la guerra ya se veía ganada, hacia 1938, la cuestión central radicaba en preparar la posguerra. Es importante rescatar el ambiente que se desarrolló en el 
Cuartel General del Generalísimo y las reflexiones que en Burgos, en las más altas esferas de poder, se llevaron a cabo durante ese período. Es necesario poner en relación los planes de ocupación con el marco temporal en que se desarrollaron, ya que no se ha estudiado el papel del orden público ni de las grandes ciudades en el modelo de su gestión. En líneas generales, tanto el pensamiento militar, su mentalidad, como la influencia que el Cuartel General pudo tener sobre la forma de llevar el conflicto y su relación con el amplio registro punitivo aparecen muy minusvalorados en la historiografía. En este sentido, la dirección de la guerra se ha entendido como instrumento político deliberado por parte de Franco para asentar su poder personal y como herramienta de eliminación masiva (Preston, 2006: 208-236 y Preston, 2008: 53-78).

Interpretar la guerra civil desde el prisma de la reacción ante la movilización social y política y su eclosión cultural, acaecida en la España urbana del primer tercio del siglo xx y entender la violencia desde una lógica funcional choca con la visión exterminista sobre los repertorios punitivos franquistas, dominante en nuestra historiografía. Una visión, todo hay que decirlo, que sigue primando el análisis desde la categoría de la víctima, lo que conduce a la descripción de todo un inventario de la brutalidad donde primaron las "atrocidades", el "terror", la "masacre" y la "purificación por la sangre". Así, desaparece la reflexión sobre la estrategia punitiva, los ritmos y cadencia de la represión o la lógica de conquista, en un escenario donde la Sevilla de julio-agosto de 1936 aparece en el mismo plano que la Cataluña de 1939. La guerra de 1936-1939 aparece definida como "guerra de aniquilación", por lo que quedarían fuera herramientas punitivas tan importantes como el sistema penitenciario, las depuraciones laborales o las jurisdicciones especiales de Responsabilidades Políticas y Masonería y Comunismo, que no tenían tal objetivo (Preston, 2011: 565-611)

Por otro lado, las estrategias de ocupación y control de los territorios controlados por la República en el verano-otoño de 1936 dominan la visión que se tiene sobre el conjunto de la guerra, lo que favorece la extensión del exterminio como categoría analítica. En este sentido, el avance de las columnas sublevadas de Sevilla a Badajoz y su actuación han dominado las explicaciones sobre el funcionamiento de la maquinaria represiva franquista, que se limita a un tiempo y a un espacio muy reducidos y se proyecta sobre el conjunto de las actuaciones. Un ejercicio que metodológicamente es contrarrestado por las cifras de asesinados en la retaguardia, que se concentran entre los meses de julio y diciembre de 1936 (Espinosa, 2003; Sevillano Calero, 2004; Moreno Gómez, 2008; Rodrigo, 2008: 42-49). Una vez más, y aunque la intención es volcarse sobre la lógica punitiva de los sublevados, la mirada se vuelve pronto hacia las víctimas como sujetos principales del análisis, cuando no se entra en un debate conceptual acerca de la conveniencia de calificar la experiencia española como "genocidio" (Eiroa, 2012; Míguez Macho, 2012 y Míguez Macho, 2014). Por último, esta secuencia interpretativa pretende vincular la sublevación y su extrema violencia con la conflictividad característica del Frente Popular, cuando sabemos que la conspiración dio sus primeros pasos mucho antes de que la obra política del Frente echara a andar (Espinosa, 2007; Puell de la Villa, 2013; Viñas, 2013).

Frente a esta visión, conviene volver la vista hacia las autoridades militares, para las que la ocupación del terreno en poder de la República era sinónimo de controlar ese mismo terreno. Es decir, volver a las lógicas que guiaron los repertorios de violencia extendidos entre 1936 y 1939, sus efectos, las bases culturales y experienciales desde las cuales partieron y los cimientos que forjaron cuando la guerra terminó y lo que siguió no fue la paz, sino el despliegue violento de la dictadura. Se hace necesario repensar la dimensión funcional de la violencia, es decir, los objetivos para los que se diseñó la compleja trama del sistema represivo franquista (Gómez Bravo y Pérez-Olivares, 2014). En este sentido, la cuestión del control social como una expresión relevante de ese sistema destaca por su escaso desarrollo en nuestra historiografía. Cuando aparece, siempre lo hace acompañada de una importante carga teórica que empaña su potencialidad práctica (Oliver, 2005; Ortiz Heras, 2010; González Calleja, 2010). Se hace necesario visibilizar este aspecto, máxime cuando en la definición de su naturaleza represiva la cuestión del control, que pone en relación la violencia con el espacio público en que se ejerce, es una de sus expresiones menos exploradas (Gómez Bravo, 2011: 11-14). Apostar por el control social como una variable explicativa de interés supone, entonces, apostar por los factores cualitativos de la violencia, por sus fundamentos socioculturales, su expresión física y la perduración de sus mecanismos en el tiempo. Supone un intento de avanzar en la comprensión de la violencia como fenómeno histórico y no como una categoría atemporal.

En las páginas anteriores se ha pretendido demostrar que la progresiva coordinación entre agencias civiles y militares, entre el espionaje militar y el Servicio de Recuperación de Documentos, entre José Ungría y Marcelino de Ulibarri, produjo una concepción muy depurada del orden público. Ésta se proyectó sobre la Columna de Orden y Policía de Ocupación, encargada de ejecutar las reflexiones de los mandos y desplegar definitivamente sus estrategias sobre el espacio. La secuencia guerraposguerra se impone, en este sentido, a otras que relacionan exclusivamente la movilización de los años republicanos (cuando no los cinco meses del Frente Popular) con la sublevación de julio de 1936. La sociedad postconflicto fue, en gran parte y al mismo tiempo, producto de la experiencia de la guerra y de la reacción contra un proceso histórico más extenso en el tiempo que la II República. La interpretación que se presenta en este artículo tiene como corolario la influencia incuestionable del Ejército en la naturaleza punitiva del régimen franquista, definido como régimen de control y coerción ya en el contexto de la propia guerra civil. No en vano fue la expresión más extendida de un régimen que apenas tuvo que recurrir al estado de excepción en sus casi cuarenta 
años de existencia, tan sólo en momentos muy concretos de crisis (Gómez Bravo, 2014). Todo ello fue ponderado en el ecuador de la guerra y proyectado sobre las grandes capitales que quedaban en territorio republicano, especialmente Madrid.

Un lugar que había sido protagonista de la movilización política de nuevos actores sociales y la eclosión de una cultura urbana que tanto asustó a ciertos sectores de la población. Éste no fue un fenómeno producido por la II República, sino que ésta fue la plasmación política de esa movilización tras el agotamiento y desborde del régimen anterior. La sublevación se produjo para poner freno a un proceso de redefinición de los límites políticos, los roles sociales y las pautas culturales. La experiencia de la guerra ayudó a configurar un modelo de orden público reactivo, apreciado por las autoridades franquistas y adaptado al mundo urbano. Madrid jugó un papel importante en todo este proceso como un objeto de deseo, el espacio donde debía plasmarse de forma prioritaria esa gestión. La documentación del Cuartel General de Franco, aún no explorada en todas sus posibilidades, permite demostrar que en los tres años de la guerra el objetivo fue tomar la capital, frente de batalla desde noviembre de 1936. Más allá de reflexiones contrafactuales sobre las consecuencias de una posible conquista en 1937 o 1938, lo relevante aquí es insistir en el papel primordial que jugaron las reflexiones del alto mando militar en la proyección del modelo de orden social para la posguerra. Para llevarlo a la práctica, la consigna estaba clara: controlar los barrios y las casas, los espacios mínimos de sociabilidad en la ciudad, mediante la coordinación de una potente red de contraespionaje y el dominio de las calles para acuñar un espacio público totalmente refractario a la Modernidad. Y Madrid, como desafío bélico y de orden público a partes iguales, fue una pieza clave en todo ese proceso.

\section{AGRADECIMIENTOS}

La propuesta de este texto se enmarca dentro de la labor del proyecto de investigación "Madrid, 1936-1939: capital, frente, retaguardia y ciudad en guerra", referencia HAR2014-52065-P. A su investigador principal, Gutmaro Gómez Bravo, agradezco la discusión de algunas cuestiones que aparecen en estas páginas.

A pesar y a través de la distancia, la reflexión sobre la ocupación y control de Madrid también es fruto de profundos debates con Daniel Oviedo Silva. La interpretación que aquí se presenta es en gran parte deudora de ellos, sobre todo en lo relativo a la importancia de la obtención de información dentro de la maquinaria punitiva franquista.

Alba Fernández Gallego me sugirió alguna de las referencias sobre la dimensión cultural de la Modernidad en la ciudad, especialmente las relacionadas con las nuevas experiencias pedagógicas. La reacción de 1936-1939 también apuntó a las ideas y configuró un orden social muy diferente al conquistado durante el primer tercio del siglo xx y representado por la II República.

\section{NOTAS}

1 Archivo General Militar de Ávila (AGMAV), Caja 1674, Carpeta 21 , p. 3.4 de noviembre de 1936. Para el avance por el sur de la península, puede consultarse Espinosa (2003).

2 Entiendo "orden público" como un concepto propio del Derecho Administrativo que incide en las nociones de seguridad, orden en sentido estricto y tranquilidad, sobre todo en su concreción en el espacio público. Frente a las posibles relaciones con términos como "legitimidad" o "consenso", este texto incide en el título de intervención que dispone la capacidad del Estado para regular sanciones y preservar la paz social. Véase Iglesias Machado (2011: 71-215). Para aproximarse al debate sobre la continuidad de la concepción tradicional de orden público, que venía del siglo xIX, véase Ballbé (1985: 398-401).

3 Citado en Preston (2006: 227).

4 "Recuperación de Documentos en la actualidad", Centro Documental de la Memoria Histórica (CDMH), DNSD-Secretaría, Expedientes personales y de asuntos, Legajo 23, Expediente 330. El subrayado es mío.

5 "Reglamento del Servicio de Recuperación de Documentos", CDMH, DNSD-Secretaría, Expedientes personales y de asuntos, Legajo 23, Expediente 330.

6 "Normas para la entrada en una ciudad ocupada", $\mathrm{CDMH}$, DNSD-Secretaría, Expedientes personales y de asuntos, Legajo 23, Expediente 330

7 "Recuperación de Documentos", CDMH, DNSD-Secretaría, Expedientes personales y de asuntos, Legajo 23, Expediente 330.

8 Ibidem.

9 "Organigrama Delegación Madrid", CDMH, DNSD, Recuperación, Secretaría, Delegación de Madrid, Caja 11, Expediente 7. El decreto de creación de la Delegación del Estado para la Recuperación de Documentos es posterior (BOE del 27 de mayo de 1938), pero el organismo ya estaba funcionando desde mediados del año anterior.

10 AGMAV, Caja 2584, Carpeta 4

11 AGMAV, Caja 2552, Carpeta 44.

12 Ibidem. El subrayado es mío.

13 Ibidem. El subrayado es mío.

14 La noción de "paisaje militarizado" hace referencia tanto a los significados asociados a los espacios como a su propia materialidad cuando entran en contacto, de una forma u otra, con la guerra. Desde la creación de espacios "artificiales" a la transformación de la naturaleza, pasando por los relatos y representaciones que vehiculan, la relación entre el paisaje y lo militar ha sido una constante histórica. Véase Pearson; Coates y Cole (2010). En el caso de este texto, se propone la adaptación del Ejército franquista al espacio urbano de Madrid como forma de refinamiento del control social.

15 En 1936 ya había sido designado delegado del Jefe de los Servicios de Ingenieros para el abastecimiento de aguas a Madrid. Véase Gorostiza y Saurí (2013). Para la importancia de la defensa de los canales de abastecimiento de agua en la defensa de Madrid, véase Gorostiza, Saurí y March (2015: 360-379).

16 Delegación para Información de Residentes en Territorio Liberado (Madrid).

17 "Para los españoles de América y de España", $A B C, 9$ de febrero de 1938. http://hemeroteca.sevilla.abc.es/nav/Navigate.exe/ hemeroteca/sevilla/abc.sevilla/1938/02/09/011.html. [Consultado el $10 / 05 / 2015]$

\section{REFERENCIAS}

Anderson, Peter (2008) "Grass-roots prosecution and collaboration in francoist military trials, 1939-1945." Contemporary European History, 18: 25-44.

Aróstegui, Julio (1996) "La defensa de Madrid y el comienzo de la guerra larga”. En La guerra de España. 1936-1939, editado por Malefakis, Edward. Taurus, Madrid: 137-166. 
Bahamonde, Ángel y Javier Cervera (2000) Así terminó la guerra de España. Marcial Pons, Madrid.

Ballbé, Manuel (1985) Orden público y militarismo en la España constitucional (1812-1983). Alianza Editorial, Madrid.

Cela, Camilo José (1951) La colmena. Emecé Editores, Buenos Aires.

Cervera, Javier (1998) Madrid en guerra. La ciudad clandestina, 1936-1939. Alianza Editorial, Madrid.

Cobo Romero, Francisco (2005) Franquismo y posguerra en Andalucía oriental. Represión, castigo a los vencidos y apoyos sociales al régimen franquista (1936-1950). Universidad de Granada, Granada.

Cruz, Rafael (1993) “Crisis del Estado y acción colectiva en el período de entreguerras, 1917-1939”. Historia Social, 15: 119-136.

Dumenil, Gérard y Dominique Lévy (2011) La crisis del neoliberalismo. Lengua de Trapo, Madrid.

Eiroa, Matilde (2012) "Prácticas genocidas en guerra, represión sistémica y reeducación social en posguerra". Hispania Nova, 10 http://hispanianova.rediris.es/10/dossier/10d014.pdf [Consultado el 10/05/2015].

Espinosa Maestre, Francisco (2003) La columna de la muerte. El avance del ejército franquista de Sevilla a Badajoz. Crítica, Barcelona.

Espinosa Maestre, Francisco (2007) La primavera del Frente Popular. Los campesinos de Badajoz y el origen de la guerra civil (marzo-julio de 1936). Crítica, Barcelona.

García Ballesteros, José Ángel y Fidel Revilla González (2006) El Madrid de la posguerra. Universidad de Mayores de Experiencia Recíproca, Madrid.

Gil Andrés, Carlos (2006) Lejos del frente. La guerra civil en la Rioja Alta. Crítica, Barcelona.

Gómez Bravo, Gutmaro (2011) "Introducción. Violencia y espacio público en la sociedad contemporánea". Cuadernos de Historia Contemporánea, 33: 11-14.

Gómez Bravo, Gutmaro (2014) Puig Antich: la Transición inacabada. Taurus, Madrid.

Gómez Bravo, Gutmaro y Jorge Marco (2011) La obra del miedo. Violencia y sociedad en la España franquista (1936-1950). Península, Barcelona.

Gómez Bravo, Gutmaro y Rubén Pallol Trigueros (2013) “Orden, delito y subversión. El estudio de la criminalidad, la conflictividad social y la violencia política en el Madrid de la primera mitad del siglo xx". En De los conflictos y sus construcciones. Mundos ibéricos y latinoamericanos, editado por Lanes, Julien Marcilhacy, David; Ralle, Michel y Rodríguez, Miguel. Editions Hispaniques, Paris: 145-163.

Gómez Bravo, Gutmaro y Alejandro Pérez-Olivares (2014) "Las lógicas de la violencia en la Guerra Civil: balance y perspectivas historiográficas". Studia Historia. Historia Contemporánea, 32: 251-262.

González Calleja, Eduardo (1998) La razón de la fuerza. Orden público, subversión y violencia política en la España de la Restauración (1875-1917). CSIC, Madrid.

González Calleja, Eduardo (1999) El máuser y el sufragio. Orden público, subversión y violencia política en la España de la Restauración (1917-1931). CSIC, Madrid.

González Calleja, Eduardo (2008) "La política de orden público en la Restauración”. Espacio, Tiempo y Forma, Serie V, t. 20: $93-$ 127

González Calleja, Eduardo (2010) "Sobre el concepto de represión". En Generaciones y memoria de la represión franquista, editado por Aróstegui, Julio y Gálvez, Sergio, . Publicacions de la Universitat de València, Valencia [CD-ROM].

González Calleja, Eduardo (2014) En nombre de la autoridad. La defensa del orden público durante la Segunda República española (1931-1936). Comares, Granada.

González Martínez, Carmen y Manuel Ortiz Heras (2010) "Control social, control policial en la dictadura franquista". Historia del Presente, 16: 143-152.

Gorostiza, Santiago y David Saurí (2013) “Salvaguardar un recurso precioso: la gestión del agua en Madrid durante la guerra civil española (1936-1939)". Scripta Nova: 457.
Gorostiza, Santiago; Hug March y David Saurí (2015) "Urban Ecology Under Fire: Water Supply in Madrid During the Spanish Civil War." Antipode, 47: 360-379.

Heiberg, Morten y Manuel Ros Agudo (2006) La trama oculta de la guerra civil. Los servicios secretos de Franco. Crítica, Barcelona.

Holguín, Sandie (2003) República de ciudadanos. Cultura e identidad nacional en la España republicana. Crítica, Barcelona.

Iglesias Machado, Salvador (2011), "La evolución del concepto de orden público y del sistema penal a través de las constituciones españolas hasta 1978". En La noción de orden público en el constitucionalismo español, editado por Delgado Aguado, Julián; Iglesias Machado, Salvador; Arnaldo Alcubilla, Enrique. Dykinson, Madrid.

Kalyvas, Stathis N. (2006) The logic of violence in civil war. Cambridge University Press, New York.

Kyre, Martin y Joan Kyre (1968) Military Occupation and National Security. Public Affairs Press, Washington D.C.

Laporta San Miguel, Francisco J.; Virgilio Zapatero; Alfonso Ruiz Miguel y Javier Solana (1987) "Los orígenes culturales de la Junta para Ampliación de Estudios". Arbor, 499-500: 9-137.

Marco, Jorge (2011) Resistencia armada en la posguerra: Andalucía oriental, 1939-1952. Universidad Complutense de Madrid. Tesis doctoral.

Martín Santos, Luis (1961) Tiempo de silencio. Seix Barral. Barcelona.

Martínez Alfaro, Encarnación (2009) Un laboratorio pedagógico de la Junta para Ampliación de Estudios. El Instituto-Escuela sección Retiro de Madrid. Biblioteca Nueva, Madrid.

Martínez Bande, José Manuel (1980) La batalla de Teruel. San Martín, Madrid.

Martínez Rus, Ana (2003) La política del libro durante la II República: socialización de la lectura. Trea, Gijón.

Melero Vargas, Miguel Ángel (2013) De la esperanza al sometimiento. Frente Popular, guerra civil y primer franquismo en una comarca andaluza. El caso de Antequera. Universidad de Málaga. Tesis Doctoral.

Míguez Macho, Antonio (2012) "Nuestro pasado presente: práctica genocida y franquismo". Hispania Nova, 10. http://hispanianova.rediris.es/10/dossier/10d014.pdf [Consultado el 10/05/2015].

Míguez Macho, Antonio (2014) La genealogía genocida del franquismo, Abada Editores, Madrid.

Montoliú, Pedro (2005) Madrid en la posguerra, 1939-1946. Los años de la represión. Sílex Ediciones, Madrid.

Moreno Gómez, Francisco (2008) 1936: el genocidio franquista en Córdoba. Crítica, Madrid.

Oliver Olmo, Pedro (2005) "El concepto de control social en la historia social: estructuración del orden y respuestas al desorden". Historia Social, 51: 73-91.

Ollaquindía, Ricardo (1995) "La Oficina de Prensa y Propaganda Carlista de Pamplona al comienzo de la guerra de 1936". Príncipe de Viana, 210: 155-170.

Otero Carvajal, Luis Enrique (2007) “Tradición y Modernidad en la España urbana de la Restauración”. En Modernizar España. Proyectos de reforma y apertura internacional (1898-1914), editado por Gómez-Ferrer, Guadalupe y Sánchez, Raquel. Biblioteca Nueva, Madrid: 79-118.

Otero Carvajal, Luis Enrique y López Sánchez, José María (2012) La lucha por la Modernidad. Las ciencias naturales y la Junta para Ampliación de Estudios. CSIC, Madrid.

Otero Carvajal, Luis Enrique y Rubén Pallol Trigueros (2009) "El Madrid moderno, capital de una España urbana en transformación, 1860-1931”. Historia Contemporánea, 39: 541-588.

Pallol Trigueros, Rubén (2009) El Madrid moderno: Chamberí (el Ensanche Norte), símbolo del nacimiento de una nueva capital, 1860-1931. Universidad Complutense de Madrid. Tesis doctoral.

Pearson, Chris; Peter Coates y Tim Cole (2010) Militarized Landscapes. From Gettysburg to Salisbury Plan. Continuum, London.

Prada, Julio (2010) La España masacrada. La represión franquista de guerra y posguerra. Alianza Editorial, Madrid.

Preston, Paul (2006) La Guerra Civil Española. Debate, Barcelona.

Preston, Paul (2008) El gran manipulador. La mentira cotidiana de Franco. Ediciones B, Barcelona. 
Preston, Paul (2011) El holocausto español. Odio y exterminio en la Guerra Civil y después. Debate, Barcelona.

Puell de la Villa, Fernando (2012) "Operaciones militares: vivencias rifeñas, lecturas de la Gran Guerra y añoranzas de Valmy". En En el combate por la historia. La República, la guerra civil y el franquismo, editado por Viñas, Ángel. Pasado y Presente, Barcelona.

Puell de la Villa, Fernando (2013) "La trama militar de la conspiración". En Los mitos del 18 de julio, coordinado por Sánchez Pérez, Francisco. Crítica, Barcelona: 55-77.

Rodrigo, Javier (2008) Hasta la raiz. Violencia durante la guerra civil y la dictadura franquista. Alianza Editorial, Madrid.

Rodríguez Martín, Nuria (2013) La capital de un sueño, Madrid 1900-1936: la formación de una metrópoli europea. Universidad Complutense de Madrid. Tesis doctoral.
Sánchez Pérez, Francisco (2005) La protesta de un pueblo. Acción colectiva y organización obrera: Madrid 1901-1923. Cinca; Fundación Largo Caballero, Madrid.

Sevillano Calero, Francisco (2004) Exterminio. El terror con Franco. Oberon, Madrid

Vicente, Fernando (2014) "Barrios negros, barrios pintorescos. Realidad e imaginario social del sub-mundo madrileño (1860-1930)". Hispania Nova, 12 (2014). http://e-revistas. uc3m.es/index.php/HISPNOV/article/view/1875/884 [Consultado 10/05/2015].

Viñas, Ángel (2013), "La connivencia fascista con la sublevación y otros éxitos de la trama civil". En Los mitos del 18 de julio, coordinado por Sánchez Pérez, Francisco. Crítica, Barcelona: $79-181$. 